Supporting Information

\title{
Reactive Molecular Dynamics Simulations of the Silanization of Silica Substrates by Methoxy- and Hydroxysilanes
}

\section{Joshua D. Deetz* ${ }^{\dagger}$, Quynh Ngo*, and Roland Faller*}

*Department of Chemical Engineering, University of California, Davis, CA 95616, United States; Contact E-mail: rfaller@ucdavis.edu

$\dagger$ Department of Chemistry and Chemical Engineering, Shanghai Jiao Tong University, Shanghai 200240, China; Contact E-mail: jddeetz@sjtu.edu.cn

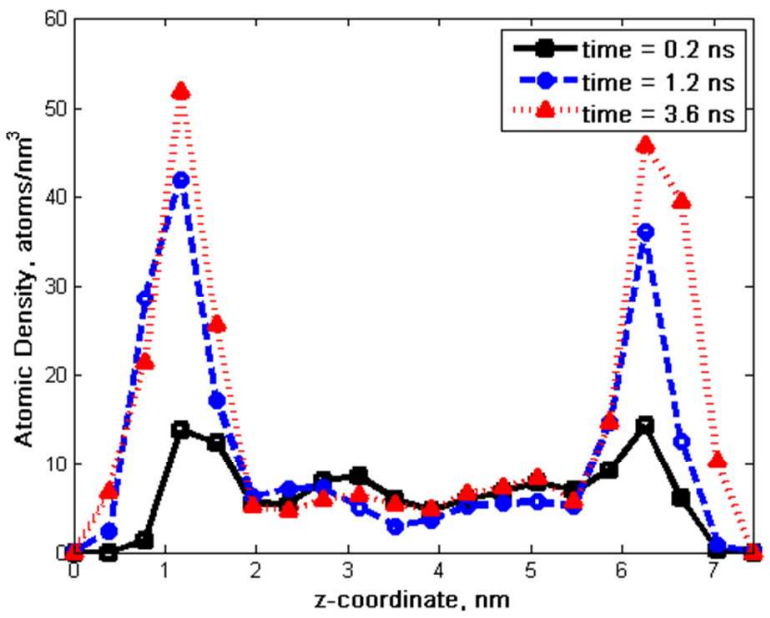

Figure S1: Atomic concentration of silane atoms in the system vs. box length and simulation time. The two silica surfaces are located approximately at 1.0 and $6.7 \mathrm{~nm}$. 


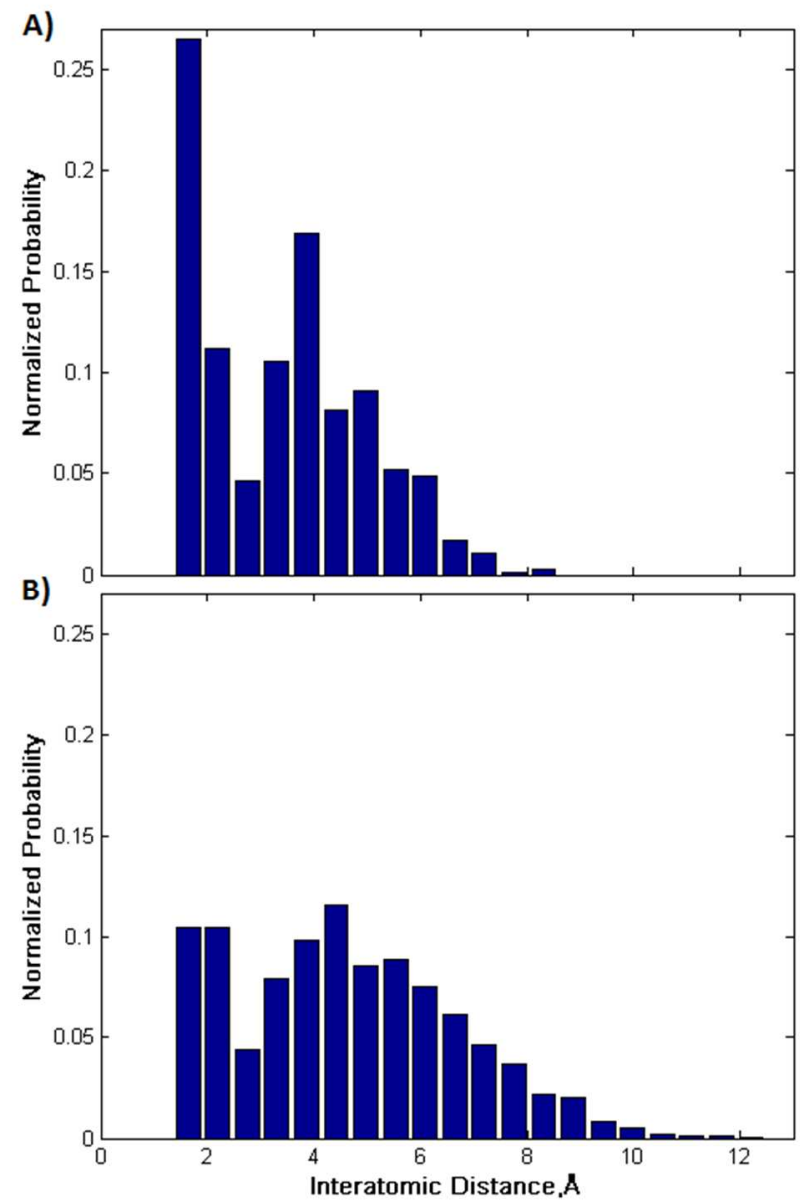

Figure S2: Normalized distributions of the closest distance between two silanol hydrogens (Si-O-H) bonded to the silica substrate. The top panel (A) shows the inter-hydrogen distance of the bare hydrolyzed substrate, whereas the bottom (B) is the averaged distribution (between BTMS, OTMS, and DTMS) after the substrate is grafted by 1.0 silanes per $\mathbf{n m}^{2}$. 